\title{
SPINAL CORD HYPOTHERMIA IN THE NEUROSURGICAL MANAGEMENT OF THE ACUTE AND CHRONIC POST-TRAUMATIC PARAPLEGIC PATIENT ${ }^{1}$
}

\author{
By JuAn Negrin, Jr., M.D., F.A.C.S., F.I.C.S., D.A.N.S. ${ }^{2}$
}

A METHOD to obtain local hypothermia of the central nervous system by extravascular perfusion of cold solutions was described by the author in I960 (Negrin, I96I). Lowering of the temperatures of the brain and spinal cord was possible with this technique which was found to be a valuable aid in clinical neurosurgery. The use of spinal cord hypothermia as an adjunct to the neurosurgical treatment of the acute and chronic traumatic paraplegic will be presented.

\section{METHOD}

The newest model of the instrument (Negrin, I970) used to obtain spinal cord hypothermia has the following parts (fig. I)

I. A container to store and distribute a cooling liquid through a tube coil system.

2. A perfusion pump to circulate the liquid at a controlled speed and pressure with a flow rate of from $25 \mathrm{ml} . / \mathrm{min}$. to $1000 \mathrm{ml} . / \mathrm{min}$.

3. A refrigeration compressor pump unit that maintains the temperature of the circulating liquid at predetermined levels.

4. A push button switch system that allows for automatic patient monitoring and temperature control of the perfusate at the operative field.

Spinal cord hypothermia is accomplished (fig. 2) by bathing pre-selected segments or the whole spinal cord with circulating cold fluids such as normal saline, Ringer's, cerebrospinal fluid, glycerol, lactate, glucose solutions, etc. In the patients to be discussed, normal saline was perfused through the subarachnoid and epidural spaces.

Post-operative cooling may be done, if necessary, for long periods without the need of local or general anesthesia, simply by reconnecting to the Hypothermostat the catheter system left properly placed at the time of surgery.

Urea, Mannitol, local, general, spinal or hypotensive anaesthesia and moderate systemic hypothermia by way of a cooling mattress, are compatible with this technique.

\section{CLINICAL OBSERVATIONS}

The following selected cases will illustrate our experience with the use of spinal cord hypothermia in the post-traumatic paraplegic.

\section{CASE REPORTS}

Case r. M. M. 45-year-old male, sustained a comminuted fracture and forward dislocation of the 4 th and 5 th thoracic vertebrae with sudden paraplegia and a correspond-

\footnotetext{
${ }^{1}$ Supported in part by the National Brain Research Foundation, U.S.A.

2 I08 East 81st Street, New York, I0028, U.S.A.
} 
ing sensory level of anaesthesia following a car accident (9.4.6I). A decompression laminectomy was done approximately five hours after the injury. The dura was not opened. Post-operative epidural cooling was done for three periods of 45 minutes each on the second, third and fourth post-operative days. No anaesthesia was used and there was full co-operation with the patient during the procedure which was carried out with



FIG. I

Hypothermostat. Instrument to obtain local hypothermia of the central nervous system (brain and spinal cord). A product of bio-medical engineering in collaboration with Swenko Research \& Development, Inc., Minneapolis, Minn., U.S.A.

the usual bedside sterile precautions. There were no complications. A complete flaccid paraplegia has persisted up to this time (about II years after injury).

Comment. This case was the first one in which we attempted to treat a traumatic spinal cord injury with local hypothermia. Since then cooling periods have been maintained for longer periods of time and continuously for up to several days when necessary. As in injuries treated with standard decompression only, the dura is never opened when it is suspected that the cord is macerated and may extrude through an opened dura under increased pressure. Cold is transmitted through the dura and the cord is cooled with a slight delay as compared with the subarachnoid technique. 
Case 2. G. L., 29-year-old male, fell down an elevator shaft (I.6.64) and sustained a comminuted compression fracture of the 7 th and 8th dorsal vertebrae and an almost complete paraplegia. There was improvement of motor power for a few hours, followed by progression of paraplegia. A decompression laminectomy (I.7.64) with combined epidural cooling two segments above and below the laminectomy and subarachnoid segmental cooling of the exposed cord were done for one hour. There was an uneventful post-operative course.



FIG. 2

Spinal cord hypothermia. Schematic representation of different techniques.

Within eight weeks the patient walked with the help of a lumbo-sacral brace only. Nine months later, over a period of eight weeks, he developed an almost complete paraplegia with urinary retention and constipation but no sensory level of hypoaesthesia. A revision of the previous laminectomy was done (I0.I I.64). A large epidural granuloma compressing the cord mainly at the level of the 7 th and 8th intervertebral space was removed. Through a $5 \mathrm{~mm}$. incision of the dura cephalid to the granuloma there was an arachnoiditis with a rich vascular plexus. No further subdural exploration was done. A thoracic fusion was carried out. There was no improvement of motor power. A few hours later, the patient developed spasticity, bilateral ankle clonus, knee clonus and painful involuntary muscle contactures of both lower extremities. Two weeks later (I0.26.64) a re-exploration was done and the dura was incised to expose the cord. The dura was 
hypertrophic. It compressed the cord reducing its width to about 20 per cent. of normal size for a length of about $3 \mathrm{~cm}$. Subarachnoid hypothermia was performed over the exposed segments and cephalid and caudally to them for one hour. The dura was not closed. The remaining surgical incision was closed in layers. Immediately the patient recovered from general anaesthesia, there was disappearance of painful muscle spasm and improvement of motor power and spasticity. A few weeks later (I2.22.64), the patient left the hospital, his sphincter control normal, walking with a back brace for support and the aid of a cane.

The patient's gait continued to improve. Six months later it became normal. At the time of his last evaluation, $5 \frac{1}{2}$ years later, he still had no neurological symptoms.

Comment. This patient is presented to illustrate what can be accomplished in acute as well as in paraplegia developing long after trauma of the cord. It also demonstrates that painful muscle spasms may be relieved after spinal cord cooling.

Case 3. J. T., I8-year-old male, was involved in a car accident (5.65) and was admitted while in a coma to St. Vincent's Hospital, Connecticut, U.S.A., where he was treated for shock and had a tracheotomy. The patient remained in coma or semicoma for about four months with spastic quadriplegia, multiple, cerebral brain stem cervical cord contusion lacerations and a fracture of the right tibia and femur. Six months after his accident he was transferred to Columbia Presbyterian Medical Centre in New York. After three weeks' evaluation he was sent to Gaylord Hospital in Connecticut. In November 1965 he was at Yale-New Haven Hospital for eight weeks and had a left motor neurotomy and tenotomy of the left semimembranous and semitendineous muscles. On 2.8.66 he was admitted to Massachusetts General Hospital where it was noted that the previous tenotomies were unsuccessful. He was advised to have a rhizotomy, which was refused, and he was then transferred to Lenox Hill Hospital, New York. At that time, the patient continued to present the original spastic quadriplegia. Passive extensions of all extremities were impossible because of extreme rigidity and pain. There was minimal voluntary extension and flexion of the right lower extremity and minimal movements of the right upper extremity. Perception of touch and pain was unreliable. He had a global aphasia and a left facial weakness. The left palpebral fissure was smaller. The X and XII cranial nerves were bilaterally involved. There were bilateral Babinski signs. The left abdominal reflexes were absent. The tracheostomy stump was open. Both trochanteric regions presented extensive inflected decubiti and a Foley catheter, which he had at various intervals following the original injury, was removed and found to be surrounded by purulent material. The fractured right tibia and femur were malunited, deformed and painful. The patient was completely bedridden and a serious nursing problem.

J. T.'s decubiti ulcers and urinary infection were treated and the tracheostomy stoma closed. A laminectomy (5.6.66) of $\mathrm{C}_{7}$ to $\mathrm{T}_{\mathrm{I}}$ was performed followed by subarachnoid cooling of the spinal cord with fluid temperatures of $7^{\circ} \mathrm{C}$. to $8^{\circ} \mathrm{C}$. perfused for 45 minutes. Intradural catheters were left in place for possible post-operative cooling. They were removed three days later because of a superficial local infection which cultured staphylococcus aureus and Gram-negative rods. A combination of staphcillin, sulpha and erythromycin controlled the infection. A left high thigh subcutaneous tenotomy was done (6.1.66) to further improve the spastic flexion adduction.

Three weeks later, the patient was able to sit in a chair and the spastic paraplegia was much improved. He began to use braces and stand up; his right upper extremity was as close to normal to the point where he could feed himself. At the time of his last evaluation, three years later, his improvement had been maintained.

Comment. This patient demonstrates the effectiveness of spinal cord hypothermia, even when traumatic lesions extend to posterior fossa and supratentorial structures. It 
also emphasises that a multidisciplinary approach, including orthopaedic procedures, may be necessary to obtain better results.

\section{DISCUSSION}

Hypothermia as a valuable adjunct of the treatment of cerebral lesions has been well established for many years (Fay, 1959). When the brain is cooled, there is a decrease in oxygen consumption and subsequent protection against anoxia during impaired or arrested circulation (Rosomoff, 1959). Futhermore, there is a decrease in brain volume (Boba, I960) and cerebrospinal fluid pressure. Cerebral oedema is decreased or arrested. Resistance of brain tissue to traumatic injuries and surgical trauma is markedly increased and surgical bleeding is easier to control.

The freezing temperatures used in cryosurgery are never reached in hypothermia. Consequently, complications secondary to freezing are avoided.

Hypothermia for the neurosurgical management of cerebral lesions has been obtained by cooling the whole body with cold baths (Longheed et al. 1955 \& Gardner et al., 1956) cooling mattresses or by intra-arterial perfusions of recirculating cold blood (Woodhall et al., 1960).

Ventricular fibrillation and cardiac standstill may appear at $90^{\circ} \mathrm{F}$. (about $32^{\circ} \mathrm{C}$.) whereas the brain may reach $4 \mathrm{I}^{\circ} \mathrm{F}$. $\left(5^{\circ} \mathrm{C}\right.$.) without permanent neurologic complication. This difference in tolerance to low temperatures leaves a large margin of low temperatures, with its inherent benefits, that cannot be used when systemic cooling is done.

In order to reach low temperature in a normothermic or moderately hypothermic body, cold capsules or ice have been applied directly to the cerebral hemispheres. Also, the arterial supply to the brain has been cooled by intra-arterial perfusion of cold blood into the cartoid system (Kristiansen \& Pott, I96I). With cold applications, including sporadic irrigation of the brain surface with cold solutions, only a slow; transitory; uncontrollable, moderate hypothermia of too small an area can be obtained. Intra-arterial perfusions to obtain systemic or local cerebral cooling require an additional major surgical procedure and increase the morbidity and mortality.

With the aim of avoiding the complications of other techniques and retaining the beneficial effects of hypothermia, the author (Negrin, I96I) developed a method that permits cerebral cooling in a normothermic body by extravascular perfusion of cold solution.

For some time now the author has noted the absence of experimental or clinical studies on the clinical potentialities of spinal cord hypothermia. It appeared to the author that hypothermia should have similar effects on spinal cord nerve tissue as on the brain and, therefore, be useful in the surgical management of lesions affecting the spinal cord. With the intention of attempting to confirm this hypothesis, and since no observations were known before the author's reports, a method to obtain local hypothermia of the spinal cord or portion of the cord in the dog was first developed (fig. 3). Even several hours after continuous hypothermia $\left(10^{\circ} \mathrm{C}\right.$. to $15^{\circ} \mathrm{C}$.) with this technique, there were no ill effects (Negrin \& Klauber, 1960).

The authors then proceeded to determine whether or not this method could have any of the practical applications surmised.

Concurrent with cardiovascular surgical research, extensive studies were carried out on the dog on the effect of systemic hypothermia in preventing neuro- 
logical sequelae following spinal cord ischaemia, produced by clamping the thoracic aorta. Local hypothermia, as described, provided protection against hind leg paralysis in the dog following experimental ischaemia, and was more effective in doing so than systemic hypothermia. There was also protection against experimental oedema and compression. That local spinal cord cooling on the dog is feasible and safe to $5^{\circ} \mathrm{C}$. was demonstrated later by other investigators (Albin et al., I96I). At this temperature there were no permanent neurological sequelae noted. In the human, we have never reached spinal cord temperatures below $5^{\circ} \mathrm{C} .\left(4 \mathrm{I}^{\circ} \mathrm{F}\right)$ and in the dog-at $15^{\circ} \mathrm{C}\left(5^{\circ} \mathrm{F}\right)$-there were no permanent neurological sequelae



FIG. 3

Experimental model to demonstrate that spinal cord hypothermia in the dog was innocuous and that spinal cord cooling prevented neurologic sequelae following experimental ischaemia, compression and oedema.

noted. This presumably indicates that there is a wide margin of safety between the hypothermic levels used in humans and the deeper ones in the dog, if it is assumed that the spinal cords in both species have a similar functional response and recovery ability to the same temperature gradients.

Local hypothermia, as used by the author, does not change the temperature of the body core or surface. Therefore, cardiac arrest and problems present in deep systemic hypothermia have not been observed.

Recent experimental studies in primates (Albin et al., I968) demonstrate that spinal cord cooling has been effective in arresting the development of severe neurological damage, due to spinal cord injury when instituted four hours after trauma. 
Prior to this study the author observed (Negrin, 1965) that spinal cord hypothermia was useful in the treatment of paraplegia of recent onset following spinal cord trauma in the human (Case 2). It protects against ischaemia, oedema and compression effects that so often complicate acute spinal cord trauma. It is also effective in certain patients with chronic traumatic paraplegia because some of their complicating symptoms such as pain, involuntary muscle contractures and muscle spasticity and rigidity may be relieved (Cases 2 and 3). Alleviation of spasticity may bring about a motor ability that may have been clinically suppressed by muscle hypertonia. There may be motor improvement to the degree where normal ambulation (Case 2) after almost complete paraplegia may be possible.

To explain the relief of muscle rigidity and spasticity following spinal cord hypothermia, the author assumes that low termperatures may produce stuctural changes of intraspinal or intradural extramedullary components of the tonic stretch reflex with a subsequent decrease in spasticity. This presupposes that there are modulators (cellular and/or fibrillar and/or synaptic and/or some or all of the elements that surround cells. fibres and synapses) capable of generating inhibitory mechanisms of the tonic stretch reflex following hypothermia.

In contraposition to this concept of newly created or modified inhibitory mechanisms or modulators, as the result of structural changes brought about by low temperatures, it is possible that these may result in a more or less complete destruction of pathways conducting excitatory stimuli to the tonic stretch reflex, with subsequent decrease or disappearance of hypertonia (Negrin, I966).

Regardless of what further experimental and clinical research may uncover regarding underlying mechanisms that relieve muscle rigidity and spasticity after spinal cord hypothermia, it has been most effective, particularly in relieving upper extremity spasticity.

There was disappearance of pain following spinal cord hypothermia in Cases 2 and 3. In I965 a similar result was noted in some cases with multiple sclerosis (Negrin, 1967). Such observations renewed our interest in the possibility of relieving intractable pain with spinal cooling. We have found this procedure an effective treatment of the type of pain often present in arthritis of the vertebral column with radicular symptoms, carcinomatosis, arachnoiditis, etc. In some instances, laminectomy can be avoided by using a simplified non-surgical technique to cool the subarachnoid space (Negrin, 1970).

Similar results with minor technical modifications have been reported recently (Hitschcock, 1967).

The relief of pain by spinal cord hypothermia may be due to a mechanism similar to the one surmised to explain the relief of muscle hypertonia. Cold may act at different sites of perception, conduction or neural integrative transmission or formation of the sensation of pain. The relief of pain by spinal cord cooling has been confirmed by other clinics.

Satisfactory neurophysiological concepts to explain these phenomena are lacking. However, the clinical results are undeniable and, in our opinion, this approach should be available in the neurosurgical management of hypertonia and pain complicating traumatic paraplegia. Its use should be strongly considered prior to other surgical techniques producing irreversible anatomical changes of the brain, spinal cord, peripheral nerves, muscles and/or tendons.

Forthcoming statistical studies will determine the percentage of results to be expected, similar to those obtained in the selected cases presented. 


\section{SUMMARY}

The technique and instrumentation to obtain local hypothermia of the spinal cord by extravascular subarachnoid or epidural perfusion of cold solutions are presented.

The usefulness of spinal cord hypothermia in the management of acute and chronic traumatic paraplegia is discussed.

Experimental observations and possible underlying neurophysiological principles are briefly reviewed.

\section{REFERENCES}

Albin, M. S., White, R. J., Acosta Rua G. \& Yashon, D. (1968). Study of functional recovery produced by delayed localised cooling after spinal cord injury in primates. f. Neurosurg, 29, I I3-I20.

Albin, M. S., White, R. J., Donald, D., MacCarthy, C. S. \& Falconer, A. Jr. (I96I). Hypothermia of the spinal cord by perfusion cooling of the subarachnoid space. Bull. Amer. Coll. Surg. Forum, 12, I88-189.

Boвa, A. (1960). Hypothemia of the neurosurgical patient. Springfield, Ill.: Ch. C. Thomas.

FAY, T. (1959). Early experiences with local generalised refrigeration of the human brain. F. Neurosurg. 16, 239-260.

GaRdNER, W. J., WASMUth, C. E. \& Hale, D. E. (1956). A method of converting an operating table into a refrigerating bath. F. Neurosurg, 13, I22-123.

HITSCHCOCK, E. (1967). Hypothermic subarachnoid irrigation for intractable pain. Lancet, $\mathbf{1 3 3}$.

KRISTIANSEN, K1. \& DotT, N. M. (I96I). Local cooling as an adjunct to cerebral operations. F. Neuro. Neuros. Psych. 24, 298.

LONGHEED, W. M. \& KAHN, D. S. (I955). Circumvention of anoxia during arrest of cerebral circulation for intracranial surgery. F. Neurosurg. 12, 226-239.

LONGHEED, W. M., SWEet, W. H., WhIte, J. C. \& BREWSTER, W. R. (I955). The use of hypothermia in the surgical treatment of cerebrovascular lesions. A preliminary report. F. Neurosurg. 12, 240-255.

NegRIN, J. Jr. (I96I). Selective local hypothermia in neurosurgery. Transactions of the 154th (1960) Annual Meeting of the Medical Society of the State of New York. N.Y.S. F. of Med. 61, 17, 2937.

NegRIN, J. Jr. (I965). Local hypothermia in spinal cord traumatic lesions. Proc. III Int. Cong. of Neurol. Surg. Copenhagen. Excerpta Medica Int., Series No. I IO, 377-381.

Negrin, J., Jr. (I966). Local hypothermia of the spincl cord for relief of spasticity and rigidity. Preliminary observations. Arch. Physical Med. Rehab. 47, I69-I 73.

NegRIN, J., Jr. (1967). Spinal cord hypothermia to relieve muscle spasticity in multiple sclerosis. Preliminary observations. F. Nerv. Ment. Dis. 144, 5, 430-434.

Negrin, J., Jr. (1970). The Hypothermostat. International Surgery, 53, 8, 93-106.

NeGRIN, J., Jr. \& KLAUBER, L. (I 959). Local hypothermia of the central nervous system. N.Y. Neur. Soc., Section of Neurology, N.Y. Ac. of Med., Proc. Arch. Neur., 3, I22, I960.

Rosomoff, H. L. (1959). Protective effects of hypothermia against pathological processes of the nervous system. Ann. N.Y. Acad. Sciences, 80, 475-486.

Woodhall, B., Sealy, W. C., Hall, K. D. \& Floyd, W. L. (I960). Craniotomy under conditions of Quinidine protected cardioplegia and profound hypothermia. Ann. Surg., 1 52, 37-44. 